\title{
Discovery of Interstellar Isocyanogen (CNCN): Further Evidence that Dicyanopolyynes Are Abundant in Space*
}

\author{
M. Agúndez (iD), N. Marcelino, and J. Cernicharo (1) \\ Instituto de Física Fundamental, CSIC, C/ Serrano 123, E-28006 Madrid, Spain; marcelino.agundez@csic.es \\ Received 2018 June 4; revised 2018 June 22; accepted 2018 June 26; published 2018 July 13
}

\begin{abstract}
It is thought that dicyanopolyynes could potentially be abundant interstellar molecules; though, their lack of a dipole moment makes it impossible to detect them through radioastronomical techniques. Recently, the simplest member of this chemical family, cyanogen $(\mathrm{NCCN})$, was indirectly probed for the first time in interstellar space through the detection of its protonated form toward the dense clouds L483 and TMC-1. Here we present a second set of firm evidence indicating the presence of NCCN in interstellar space, namely the detection of the metastable and polar isomer isocyanogen $(\mathrm{CNCN})$. This species has been identified in $\mathrm{L} 483$ and tentatively in TMC-1 by observing various rotational transitions in the $\lambda 3 \mathrm{~mm}$ band with the IRAM $30 \mathrm{~m}$ telescope. We derive beamaveraged column densities for CNCN of $1.6 \times 10^{12} \mathrm{~cm}^{-2}$ in L483 and $9 \times 10^{11} \mathrm{~cm}^{-2}$ in TMC-1, which imply fractional abundances relative to $\mathrm{H}_{2}$ in the range of $(5-9) \times 10^{-11}$. While the presence of NCCN in interstellar clouds seems undoubtable due to the detection of $\mathrm{NCCNH}^{+}$and $\mathrm{CNCN}$, putting tight constraints on its abundance is still hampered by the poor knowledge of the chemistry that links $\mathrm{NCCN}$ with $\mathrm{NCCNH}^{+}$and especially with CNCN. We estimate that NCCN could be fairly abundant, in the range of $10^{-9}-10^{-7}$ relative to $\mathrm{H}_{2}$, such as other abundant nitriles like $\mathrm{HCN}$ and $\mathrm{HC}_{3} \mathrm{~N}$.
\end{abstract}

Key words: astrochemistry - ISM: clouds - ISM: molecules - line: identification - radio lines: ISM

Supporting material: data behind figures

\section{Introduction}

Interstellar chemistry is essentially organic in nature. Around three-fourths of the nearly 200 molecules detected to date in interstellar and circumstellar media contain at least one carbon atom. Among them, there are alcohols, aldehydes, acids, ethers, and amines; however, the most prevalent functional group is that of nitriles (e.g., Agúndez \& Wakelam 2013). Indeed, the strong bond of the cyano group $-\mathrm{C} \equiv \mathrm{N}$ is present in more than 30 interstellar molecules, though until recently, no molecule containing two cyano groups had been observed in interstellar space (Agúndez et al. 2015a).

A particular subclass of molecules with two cyano groups are dicyanopolyynes, which consist of a highly unsaturated linear skeleton of carbon atoms ended by a cyano group at each edge, i.e., $\mathrm{N} \equiv \mathrm{C}-(\mathrm{C} \equiv \mathrm{C})_{n}-\mathrm{C} \equiv \mathrm{N}$. These molecules are very stable and it has been suggested that they could be abundant in interstellar and circumstellar clouds (Kołos \& Grabowski 2000; Petrie et al. 2003). The simplest member of this type of molecules, cyanogen (NCCN), is hypothesized to be a precursor of the widely observed cometary $\mathrm{CN}$ (Fray et al. 2005) and it has been identified in the atmosphere of Titan (Kunde et al. 1981), where the larger homologue $\mathrm{NC}_{4} \mathrm{~N}$ has been long thought to be present as well (Jolly et al. 2015). A chemical cousin of cyanogen in which one $\mathrm{N}$ atom is substituted by a $\mathrm{P}$ atom, NCCP, has been tentatively identified in the carbon-rich envelope IRC+10216 (Agúndez et al. 2014).

It is therefore reasonable to think that dicyanopolyynes can be abundant in molecular clouds. However, their detection is hampered by the fact that they are nonpolar and therefore cannot be observed through their rotational spectrum. To

\footnotetext{
* Based on observations carried out with the IRAM $30 \mathrm{~m}$ Telescope. IRAM is supported by INSU/CNRS (France), MPG (Germany), and IGN (Spain).
}

investigate the plausibility of this hypothesis, it was proposed that the presence of NCCN in interstellar and circumstellar clouds can be indirectly probed through the observation of chemically related polar molecules, such as protonated cyanogen $\left(\mathrm{NCCNH}^{+}\right)$or the metastable isomer $\mathrm{CNCN}$ (Petrie et al. 2003). A few years ago, we succeeded in detecting protonated cyanogen in the cold dark clouds L483 and TMC- 1 (Agúndez et al. 2015a). From the observed abundances of $\mathrm{NCCNH}^{+}$it was inferred that indeed NCCN should be as abundant as the nitriles $\mathrm{HCN}$ and $\mathrm{HC}_{3} \mathrm{~N}$. In this Letter, we present the identification of isocyanogen $(\mathrm{CNCN})$, a metastable isomer of $\mathrm{NCCN}$, in $\mathrm{L} 483$ and a tentative detection toward TMC-1. This result brings further support to the hypothesis that dicyanopolyynes are abundant molecules in interstellar space.

\section{Observations}

The observations of L483 were carried out with the IRAM $30 \mathrm{~m}$ telescope in the course of a $\lambda 3 \mathrm{~mm}$ molecular line survey. Details of these observations are given in Agúndez et al. (2018) and Marcelino et al. (2018), while a thorough description of the complete survey will be presented elsewhere (M. Agúndez et al. 2018, in preparation). Briefly, observations were performed in several sessions from 2016 August to 2018 April, with the telescope pointed toward the position of the infrared source IRAS 18148-0440. We used the frequencyswitching technique, with the receiver EMIR E090 connected to the FTS backend with a spectral resolution of $50 \mathrm{kHz}$.

The observations of TMC-1 were also taken with the IRAM $30 \mathrm{~m}$ telescope as part of a $\lambda 3 \mathrm{~mm}$ spectral scan at the position of the cyanopolyyne peak (Marcelino et al. 2005, 2007, 2009). A large fraction of the data was observed in 2012 February (see details in Cernicharo et al. 2012). 


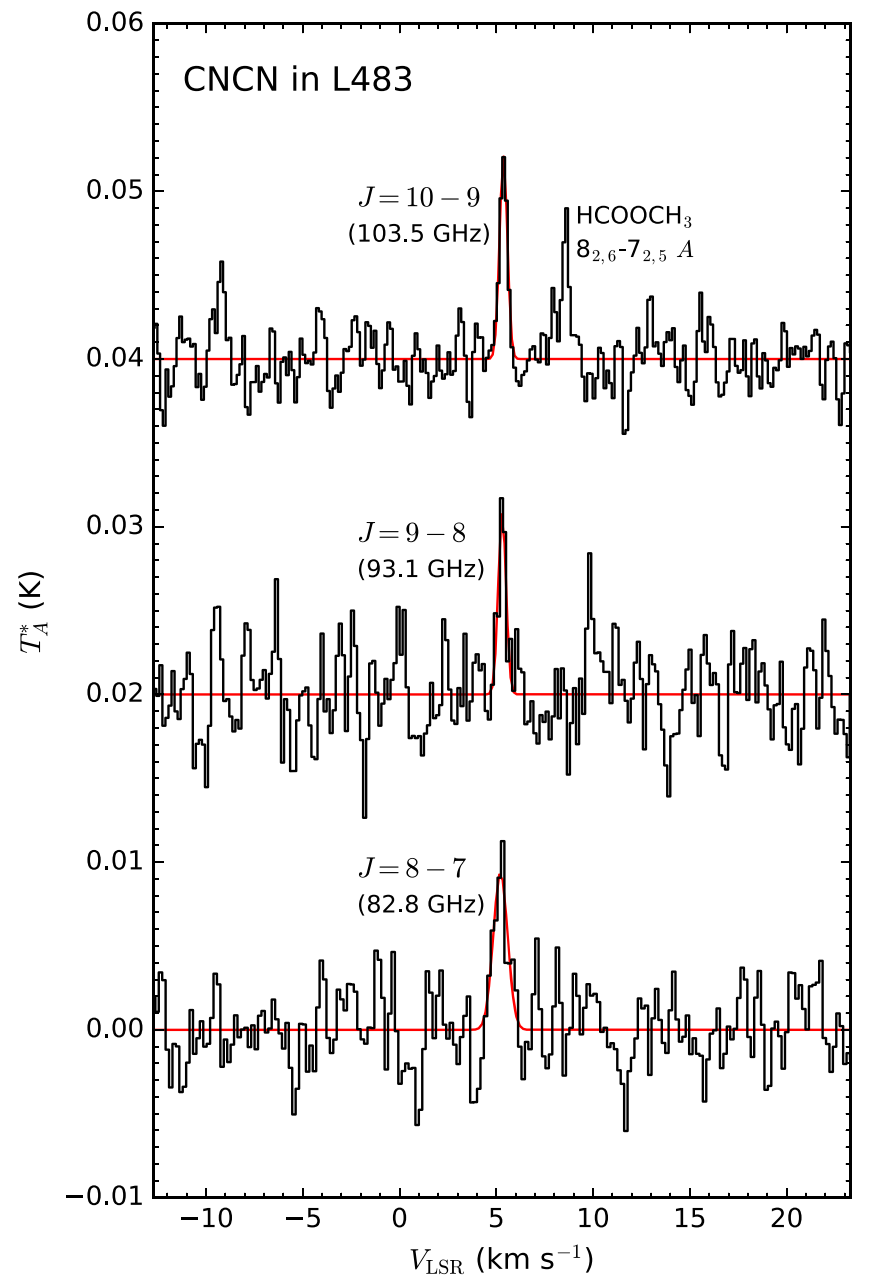

Figure 1. Lines of CNCN observed toward L483 with the IRAM $30 \mathrm{~m}$ telescope. Lines are detected at $8-14 \sigma$ confidence levels. The $J=11-10$ line at $113.8 \mathrm{GHz}$ is not detected due to the more limited sensitivity of the data at these frequencies. The data used to create this figure are available.

\section{Results}

The analysis of the $\lambda 3 \mathrm{~mm}$ IRAM $30 \mathrm{~m}$ data of L483 has already resulted in the detection of several new interstellar molecules: HCCO (Agúndez et al. 2015b), $\mathrm{NCCNH}^{+}$(Agúndez et al. 2015a), $\mathrm{NS}^{+}$(Cernicharo et al. 2018), HCS, and HSC (Agúndez et al. 2018), as well as NCO and the confirmation of $\mathrm{H}_{2} \mathrm{NCO}^{+}$(Marcelino et al. 2018). A complete analysis of the $\lambda 3 \mathrm{~mm}$ molecular line survey will be presented in a separate article (M. Agúndez et al. 2018, in preparation). Here we focus on three emission lines that can be unambiguously assigned to the $J=8-7, J=9-8$, and $J=10-9$ rotational transitions of $\mathrm{CNCN}$, lying at $82.8 \mathrm{GHz}, 93.1 \mathrm{GHz}$, and $103.5 \mathrm{GHz}$ (see Figure 1 and Table 1).

Isocyanogen $(\mathrm{CNCN})$, a metastable isomer lying $1.06 \mathrm{eV}$ above cyanogen $(\mathrm{NCCN})$, is a linear molecule with a ${ }^{1} \Sigma^{+}$ electronic ground state (Botschwina \& Sebald 1990; Ding et al. 2000; Chaudhuri et al. 2006). Its rotational spectrum has been extensively characterized in the laboratory from 70 to $340 \mathrm{GHz}$ (Winnewisser et al. 1992). The hyperfine structure due to the quadrupole of the two ${ }^{14} \mathrm{~N}$ nuclei has been measured for the $J=1-0$ and $J=2-1$ lines, where the various components are separated by a few MHz (Gerry et al. 1990). However, the higher- $J$ lines observed here have very small line

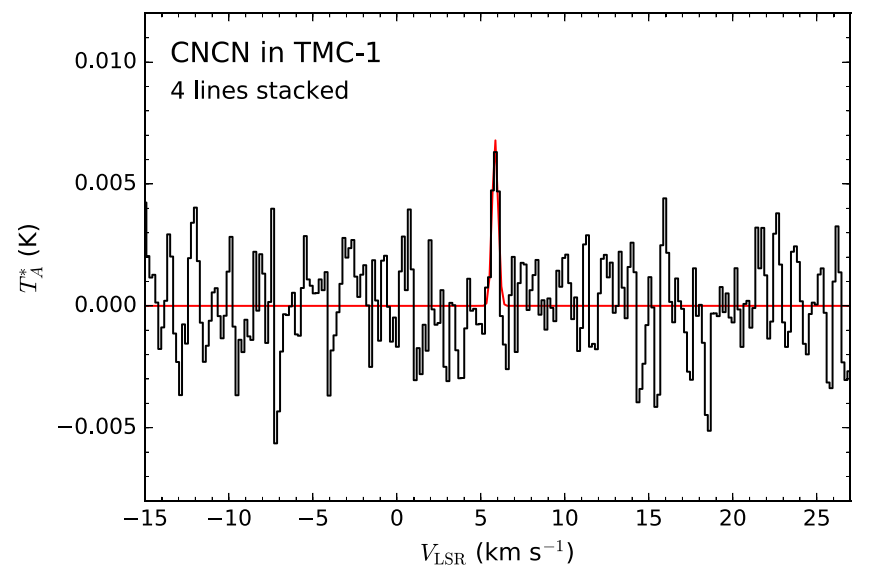

Figure 2. IRAM $30 \mathrm{~m}$ spectrum obtained toward TMC-1 by stacking four lines, the $J=8-7$ through $J=11-10$, of CNCN. The line is detected at a $5-6 \sigma$ confidence level. The data used to create this figure are available.

Table 1

Observed Line Parameters of CNCN in L483

\begin{tabular}{lcccc}
\hline \hline Transition & $\begin{array}{c}\text { Frequency } \\
(\mathrm{MHz})\end{array}$ & $\begin{array}{c}V_{\mathrm{LSR}} \\
\left(\mathrm{km} \mathrm{s}^{-1}\right)\end{array}$ & $\begin{array}{c}\Delta v \\
\left(\mathrm{~km} \mathrm{~s}^{-1}\right)\end{array}$ & $\begin{array}{c}\int T_{A}^{*} d v \\
\left(\mathrm{~K} \mathrm{~km} \mathrm{~s}^{-1}\right)\end{array}$ \\
\hline$J=8-7$ & 82784.692 & $+5.21(7)$ & $0.87(14)$ & $0.0087(13)$ \\
$J=9-8$ & 93132.326 & $+5.30(4)$ & $0.47(14)$ & $0.0052(4)$ \\
$J=10-9$ & 103479.802 & $+5.37(3)$ & $0.47(6)$ & $0.0061(6)$
\end{tabular}

Note. Numbers in parentheses are $1 \sigma$ uncertainties in units of the last digits obtained from Gaussian fits.

splittings and cannot be spectrally resolved (see JPL catalog ${ }^{1}$; Pickett et al. 1998). Isocyanogen is a moderately polar molecule, with a measured permanent electric dipole moment of $0.7074 \pm 0.0052 \mathrm{D}$ (Gerry et al. 1990). The species is included in the MADEX code $^{2}$ (Cernicharo 2012).

The spectra of L483 at 82.8 and $93.1 \mathrm{GHz}$ have $T_{A}^{*}$ rms noise levels of $2.2-2.3 \mathrm{mK}$ per $50 \mathrm{kHz}$ channel, which translates to detection confidence levels of $8-9 \sigma$ for the $J=8-7$ and $J=9-8$ lines of CNCN. We note that the Gaussian fit to the $J=8-7$ line results in a too large line width $\left(0.87 \mathrm{~km} \mathrm{~s}^{-1}\right.$ versus the value of $0.47 \mathrm{~km} \mathrm{~s}^{-1}$ obtained for the two other lines; see Table 1) which is probably a consequence of the presence of positive channels around the line which are within the noise but are taken as part of the line by the fitting algorithm. The spectrum at $103.5 \mathrm{GHz}$ is more sensitive, with a $T_{A}^{*} \mathrm{rms}$ of $1.6 \mathrm{mK}$ per $50 \mathrm{kHz}$ channel, and the $J=10-9$ line is detected at a higher confidence level of $14 \sigma$. This spectrum also clearly shows a weak line arising from methyl formate (see Figure 1). The line survey indeed shows many more lines of this complex organic molecule, which has recently been detected in L483 using ALMA (Oya et al. 2017). There is another rotational transition of $\mathrm{CNCN}$, the $J=11-10$ lying at $113.8 \mathrm{GHz}$, which falls within the frequency range covered by our line survey; though, in this spectral region the data is less sensitive (the $T_{A}^{*} \mathrm{rms}$ noise level is $\sim 5 \mathrm{mK}$ per $50 \mathrm{kHz}$ channel) and the line is not clearly detected. With three lines well detected, we consider that the identification of $\mathrm{CNCN}$ in L483 is completely secure.

\footnotetext{
https://spec.jpl.nasa.gov/

2 https://nanocosmos.iff.csic.es/?page_id=1619
} 
We have also searched for $\mathrm{CNCN}$ in our $\lambda 3 \mathrm{~mm}$ data of TMC-1 obtained with the IRAM $30 \mathrm{~m}$ telescope. The data covers the four rotational transitions, $J=8-7$ through $J=11-10$. None of them are detected at a sufficient confidence level, but after stacking the spectra around the four lines, an emission line with a peak intensity of $T_{A}^{*} \sim 7 \mathrm{mK}$ appears centered at $V_{\mathrm{LSR}}$ of $5.85 \pm 0.05 \mathrm{~km} \mathrm{~s}^{-1}$, in agreement with the systemic velocity of TMC-1 measured in other molecular lines (e.g., Agúndez et al. 2015a). The line is detected at a 5-6 $\sigma$ confidence level. However, taking into account that it comes from a line stacking of four lines, we consider the detection to be tentative. More sensitive observations are needed to detect the individual lines with a sufficient signal-to-noise ratio.

From the intensities of the three $\mathrm{CNCN}$ lines detected in L483 and assuming a rotational temperature of $10 \mathrm{~K}$, which is the gas kinetic temperature in this dense core as derived from $\mathrm{NH}_{3}$ (Anglada et al. 1997), we find a beam-averaged column density for CNCN of $1.6 \times 10^{12} \mathrm{~cm}^{-2}$. In TMC-1, we estimate $N(\mathrm{CNCN})=9 \times 10^{11} \mathrm{~cm}^{-2}$, assuming a rotational temperature of $10 \mathrm{~K}$ (Pratap et al. 1997). Adopting $\mathrm{H}_{2}$ column densities of $3 \times 10^{22} \mathrm{~cm}^{-2}$ for L483 (Tafalla et al. 2000) and $1 \times 10^{22} \mathrm{~cm}^{-2}$ for TMC-1 (Cernicharo \& Guélin 1987), the abundance of CNCN relative to $\mathrm{H}_{2}$ is then $5 \times 10^{-11}$ in $\mathrm{L} 483$ and $9 \times 10^{-11}$ in TMC-1. The metastable isomer CNCN is therefore significantly more abundant than protonated cyanogen, with column density ratios $\mathrm{CNCN} / \mathrm{NCCNH}^{+}$of 40 and 10 in L483 and TMC-1, respectively.

The dense cores TMC-1 and L483 are rich in carbon chain molecules (e.g., Kawaguchi et al. 1995; M. Agúndez et al. 2018, in preparation), and this common feature may be at the origin of the presence of cyanogen in both clouds. The two sources are, however, different in that TMC- 1 is a starless core that displays narrow lines (Soma et al. 2018), while L483 hosts a Class 0 protostar and some molecules show wide lines for which part of the emission arises from an outflow (e.g., $\mathrm{H}_{2} \mathrm{CO}$; Tafalla et al. 2000) or from a compact hot corino around the protostar, as revealed by recent ALMA observations (e.g., HNCO; Oya et al. 2017). The narrow line profiles of $\mathrm{CNCN}$ in $\mathrm{L} 483$ point to emission from the ambient cloud. We estimate a $3 \sigma$ upper limit to the abundance of $\mathrm{CNCN}$ in the hot corino of (5-7) $\times 10^{-9}$ relative to $\mathrm{H}_{2}$, adopting a source diameter of 0 ". 5 , a line width of $10 \mathrm{~km} \mathrm{~s}^{-1}$, a rotational temperature of $70-130 \mathrm{~K}$, and $N\left(\mathrm{H}_{2}\right)=6.5 \times 10^{23} \mathrm{~cm}^{-2}$ (see Oya et al. 2017).

\section{Discussion}

The discovery of $\mathrm{CNCN}$ opens a new venue, in addition to that provided by $\mathrm{NCCNH}^{+}$, to probe the presence of the stable and likely abundant, though nonpolar, cyanogen molecule (NCCN). However, in order to use these species as markers of cyanogen it is necessary to understand the underlying chemical relationships between them.

The chemistry of $\mathrm{NCCNH}^{+}$and its chemical connection with NCCN was studied by Petrie et al. (2003) and more recently by Agúndez et al. (2015a). In both studies, the $\mathrm{NCCNH}^{+} / \mathrm{NCCN}$ abundance ratio predicted from a cold dark cloud chemical model is of the order of $10^{-4}$. Therefore, the observed abundances of $\mathrm{NCCNH}^{+}$put the abundance of NCCN in dark clouds at the level of $(1-10) \times 10^{-8}$ relative to $\mathrm{H}_{2}$ (Agúndez et al. 2015a). We note, however, that in this latter study the observed abundance ratios between the protonated and nonprotonated forms of the related cyanides HCN (and
$\mathrm{HNC}$ ) and $\mathrm{HC}_{3} \mathrm{~N}$ are underestimated by the chemical model by roughly a factor of 10 . If the same holds for NCCN, its abundance would need to be revised downward to $(1-10) \times 10^{-9}$ relative to $\mathrm{H}_{2}$.

In the case of the isomer CNCN, little is known about its chemistry and thus it is difficult to relate the observed abundance to that of NCCN. However, as noted by Petrie et al. (2003), this pair of isomers share structural characteristics (namely, a $-\mathrm{CNC}$ versus a $-\mathrm{CCN}$ subunit) with $\mathrm{HCCNC}$ and HCCCN, both of which are observed in cold dark clouds. Concretely, in L483 and TMC-1 the HCCNC/HCCCN column density ratio is around 50 (details for L483 will follow in M. Agúndez et al. 2018, in preparation; see, e.g., Agúndez \& Wakelam 2013 for TMC-1). If the CNCN/NCCN abundance ratio is similar, the observed abundances of $\mathrm{CNCN}$ in $\mathrm{L} 483$ and TMC-1 would imply an NCCN abundance of (2.5-4.5) $\times$ $10^{-9}$ relative to $\mathrm{H}_{2}$. It is worth noting that these values are in the range of abundances inferred above from $\mathrm{NCCNH}^{+}$ adopting an $\mathrm{NCCNH}^{+} / \mathrm{NCCN}$ abundance ratio of $10^{-3}$, instead of $10^{-4}$.

In any case, it is clear that a better understanding of the chemistry of $\mathrm{NCCN}, \mathrm{CNCN}$, and $\mathrm{NCCNH}^{+}$in interstellar clouds is necessary to draw more robust conclusions. It is therefore worth revisiting what is known and in which directions further research is needed.

It is well established that the reaction between $\mathrm{CN}$ and $\mathrm{HCN}$, which is exothermic to yield cyanogen, has an important activation barrier that makes it too slow at low temperatures (Yang et al. 1992). Therefore, the most plausible formation route of NCCN in cold clouds is the reaction

$$
\begin{aligned}
\mathrm{CN}+ & \mathrm{HNC} \rightarrow \mathrm{NCCN}+\mathrm{H}, \\
& \rightarrow \mathrm{CNCN}+\mathrm{H},
\end{aligned}
$$

which involves two abundant reactants and is barrierless to produce NCCN, though, not when forming $\mathrm{CNCN}$ (Petrie et al. 2003). It would nevertheless be interesting to revisit this reaction through experiments or dynamical calculations to quantify the kinetics of the various possible channels. It is difficult to envisage other feasible (rapid and involving abundant reactants) NCCN-forming reactions. Another possible route is the reaction between $\mathrm{N}$ and $\mathrm{HCCN}$, for which Loison et al. (2015) estimate a moderate rate coefficient of a few times $10^{-11} \mathrm{~cm}^{3} \mathrm{~s}^{-1}$; though, this reaction would be less efficient than reaction 1(a) owing to the low abundance of HCCN. Reactions of $\mathrm{N}_{2}$ with radicals are unlikely to be exothermic due to the strong triple bond of molecular nitrogen. For example, the reaction between $\mathrm{N}_{2}$ and $\mathrm{C}_{2} \mathrm{H}$ to produce $\mathrm{NCCN}$ is endothermic by $\sim 50 \mathrm{~kJ} \mathrm{~mol}^{-1}$.

If neutral-neutral gas-phase routes are not efficient enough, routes involving ions may do the work. The most obvious is

$$
\mathrm{NCCNH}^{+}+\mathrm{e}^{-} \rightarrow \text { products }
$$

which could reasonably lead to $\mathrm{NCCN}+\mathrm{H}$ or $\mathrm{CNCN}+\mathrm{H}$, although neither the rate coefficient nor the product distribution have been measured. A similar mechanism involving the ion $\mathrm{HCNH}^{+}$is thought to form $\mathrm{HCN}$ and HNC in dark clouds (e.g., Agúndez \& Wakelam 2013). Note that if $\mathrm{NCCNH}^{+}$is the precursor of NCCN, and not the reverse, as occurs if NCCN is mainly formed by neutral-neutral routes like reaction 1(a), then the $\mathrm{NCCNH}^{+} / \mathrm{NCCN}$ abundance ratio will no longer be of the 
order of $10^{-4}$, but significantly higher. The question then would be how can $\mathrm{NCCNH}^{+}$be efficiently formed, typically from smaller fragments. One possible candidate is

$$
\mathrm{HCNH}^{+}+\mathrm{CN} \rightarrow \mathrm{NCCNH}^{+}+\mathrm{H},
$$

which is a potentially important source of $\mathrm{NCCNH}^{+}$because the reactants are relatively abundant. Reaction (3) is nearly thermoneutral and thus it is unclear whether or not it is rapid at low temperatures. The reaction between $\mathrm{HCN}^{+}$and $\mathrm{HCN}$ is exothermic in the channel yielding $\mathrm{NCCNH}^{+}$. However, the main channel appears to be $\mathrm{HCNH}^{+}+\mathrm{CN}$ (Huntress et al. 1969) and besides, the reactant $\mathrm{HCN}^{+}$should not be very abundant in dark clouds. Other potentially interesting reactions forming $\mathrm{NCCNH}^{+}$are $\mathrm{C}_{2} \mathrm{~N}^{+}+\mathrm{NH}_{2}$ and $\mathrm{C}_{2} \mathrm{NH}^{+}+\mathrm{NH}$; though, the reactants are probably not abundant enough in dark clouds. The isomer $\mathrm{CNCN}$ can be formed in the dissociative recombination of $\mathrm{NCCNH}^{+}$with electrons if some important rearrangement takes place. A study of the product distribution of reaction (2) would allow us to validate or refute this possibility. If $\mathrm{NCCNH}^{+}$is not a viable precursor for $\mathrm{CNCN}$, then protonated isocyanogen (either $\mathrm{HCNCN}^{+}$or $\mathrm{CNCNH}^{+}$, both having similar energies; Petrie 1998; Ding et al. 2001) could be the main precursor. However, protonated isocyanogen is $60-65 \mathrm{~kJ} \mathrm{~mol}^{-1}$ less stable than protonated cyanogen (Petrie 1998) and thus forming it would be more difficult. For example, while a priori reaction (3) is a viable route to $\mathrm{NCCNH}^{+}$, the channel leading to $\mathrm{HCNCN}^{+}$or $\mathrm{CNCNH}^{+}$is probably endothermic.

A synthetic route to $\mathrm{NCCN}$ and $\mathrm{CNCN}$ alternative to the gas phase could take place on dust grains by recombination of two $\mathrm{CN}$ radicals. It is, however, uncertain whether the abundance of $\mathrm{CN}$ on grain surfaces is high enough. The $\mathrm{CN}$ radical, as other nitrogen-bearing species, tend to show little depletion from the gas phase in dense cores (Hily-Blant et al. 2008). It is also not clear whether such a reaction may have some barrier and ultimately how effective the desorption of these species would be for the gas phase.

Whatever the mechanism of formation of NCCN, it is likely to have a long life time in cold dark clouds because it is not a very reactive species, although the same may not be true for CNCN. Cyanogen shows a moderate reactivity with ions (McEwan \& Anicich 1995) and it does not react at low temperature with $\mathrm{H}$ or $\mathrm{O}$ atoms (see NIST database ${ }^{3}$ ). However, it reacts with $\mathrm{C}$ and $\mathrm{N}$ atoms

$$
\begin{aligned}
& \mathrm{NCCN}+\mathrm{C} \rightarrow \mathrm{CN}+\mathrm{C}_{2} \mathrm{~N}, \\
& \mathrm{NCCN}+\mathrm{N} \rightarrow \mathrm{N}_{2}+\mathrm{C}_{2} \mathrm{~N},
\end{aligned}
$$

with rate coefficients of a few times $10^{-11} \mathrm{~cm}^{3} \mathrm{~s}^{-1}$ at room temperature (Safrany \& Jaster 1968; Whyte \& Phillips 1983). However, taking into account that reactions (4) and (5) are thought to be the main depletion mechanisms of NCCN (Agúndez et al. 2015a), it would be interesting to study their low-temperature kinetics.

\section{Prospects}

There are various directions to advance in our understanding of the presence and abundance of cyanogen and dicyanopolyynes

\footnotetext{
https://kinetics.nist.gov/kinetics/
}

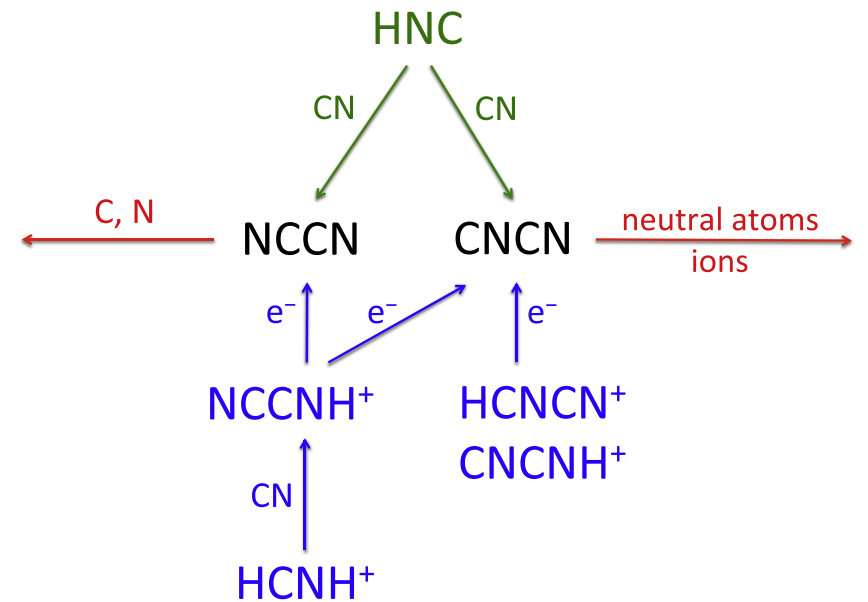

Figure 3. Main formation (neutral in green and ionic in blue) and destruction (in red) pathways for NCCN and CNCN. The low-temperature kinetics of these reactions is very poorly constrained.

in interstellar space. The first one would be to study reactions (1)-(5) either experimentally or theoretically (see Figure 3) to better understand the chemical relationship of $\mathrm{NCCNH}^{+}$and CNCN with the likely abundant but unobservable NCCN.

The astronomical search for other markers of cyanogen is also an interesting possibility. For example, apart from CNCN, there are another two linear metastable isomers of NCCN. These are di-isocyanogen (CNNC) and diaza-dicarbon (NNCC), isomeric forms that lie higher in energy, specifically at 3.16 and $3.90 \mathrm{eV}$, respectively, above NCCN (Ding et al. 2000; Chaudhuri et al. 2006). While CNNC is nonpolar, NNCC is calculated to have a large dipole moment of $3.35 \mathrm{D}$ (Ding et al. 2000), and it is thus a candidate for interstellar detection. This isomer, however, seems elusive to laboratory detection (Maier et al. 1992) and therefore its rotational spectrum remains unknown. An astronomical search for the protonated form of $\mathrm{CNCN}$, either $\mathrm{HCNCN}^{+}$or $\mathrm{CNCNH}^{+}$(Petrie 1998; Ding et al. 2001), would also provide valuable information on the chemical origin of $\mathrm{CNCN}$, but the rotational spectrum has not been measured.

If cyanogen is really an abundant species in interstellar clouds, it is conceivable that larger members of the series of dicyanopolyynes are present as well. The next member is dicyanoacetylene $\left(\mathrm{NC}_{4} \mathrm{~N}\right)$, which, as $\mathrm{NCCN}$, is nonpolar and thus indirect strategies to probe it are necessary. Dicyanoacetylene can be efficiently formed through the reactions $\mathrm{CN}+\mathrm{HC}_{3} \mathrm{~N}$ or $\mathrm{C}_{3} \mathrm{~N}+\mathrm{HCN} / \mathrm{HNC}$, which are barrierless according to calculations by Petrie \& Osamura (2004). An astronomical search for the protonated form of $\mathrm{NC}_{4} \mathrm{~N}$ is not yet feasible because there is little spectroscopic information on this ion. However, the metastable isomer $\mathrm{NC}_{3} \mathrm{NC}$, which lies $1.12 \mathrm{eV}$ above $\mathrm{NC}_{4} \mathrm{~N}$ has a moderate calculated dipole moment of 1.04-1.096 D (Horn et al. 1994; Lee 1998), and its rotational spectrum has been measured in the laboratory (Huckauf et al. 1999). It is not present in our $\lambda 3 \mathrm{~mm}$ data of L483 and TMC-1; though, this is probably not the best wavelength range to search for such a heavy molecule in cold sources. A search at longer wavelengths may prove more fruitful. There are another two metastable isomers, but one $\left(\mathrm{CNC}_{2} \mathrm{NC}\right)$ is nonpolar while for the other $\left(\mathrm{NCNC}_{3}\right.$ ), which lies $2.60 \mathrm{eV}$ above $\mathrm{NC}_{4} \mathrm{~N}$ (Jiang et al. 2004), spectroscopic data is lacking. 
A better understanding of the chemistry of NCCN in interstellar space would benefit from the search for the already detected species $\mathrm{NCCNH}^{+}$and $\mathrm{CNCN}$ in other sources, ranging from prestellar cores to star-forming regions. Getting access to the spatial distribution of these species in the targeted clouds will also help; though, lines are weak and obtaining emission maps will be difficult, especially if NCCN and their markers $\mathrm{NCCNH}^{+}$and $\mathrm{CNCN}$ have an extended distribution over the ambient cloud, which is the most likely possibility. Finally, a direct search for NCCN at infrared wavelengths with telescopes like SOFIA or JWST may prove to be fruitful for strong infrared sources.

\section{Conclusions}

We have reported on the first identification in space of isocyanogen $(\mathrm{CNCN})$, a metastable isomer of cyanogen. This species has been observed in the dense core L483 and tentatively in TMC-1, where we previously reported the detection of protonated cyanogen $\left(\mathrm{NCCNH}^{+}\right)$. We derive fractional abundances of several times $10^{-11}$ relative to $\mathrm{H}_{2}$ for CNCN. The detection of $\mathrm{CNCN}$ together with that of $\mathrm{NCCNH}^{+}$in dark clouds supports the idea that NCCN and larger dicyanopolyynes, all of which are nonpolar and thus cannot be directly observed through their rotational spectrum, are abundant in molecular clouds. We estimate that the abundance of NCCN may be in the range of $10^{-9}-10^{-7}$ relative to $\mathrm{H}_{2}$. A better knowledge of the chemistry of NCCN and dicyanopolyynes in space will benefit from experimental and/or theoretical studies of some key reactions together with sensitive astronomical observations of $\mathrm{NCCNH}^{+}, \mathrm{CNCN}$, and related species not yet identified in space.

We thank the IRAM $30 \mathrm{~m}$ staff for their help during the observations and the anonymous referee for useful comments. We acknowledge funding support from the European Research Council (ERC Grant 610256: NANOCOSMOS) and from Spanish MINECO through grant AYA2016-75066-C2-1-P. M.A. also acknowledges funding support from the Ramón y Cajal programme of Spanish MINECO (RyC-2014-16277).

\section{ORCID iDs}

M. Agúndez (iD https://orcid.org/0000-0003-3248-3564

J. Cernicharo (i) https://orcid.org/0000-0002-3518-2524

\section{References}

Agúndez, M., Cernicharo, J., de Vicente, P., et al. 2015a, A\&A, 579, L10 Agúndez, M., Cernicharo, J., \& Guélin, M. 2014, A\&A, 570, A45 Agúndez, M., Cernicharo, J., \& Guélin, M. 2015b, A\&A, 577, L5

Agúndez, M., Marcelino, N., Cernicharo, J., \& Tafalla, M. 2018, A\&A, 611, L1 Agúndez, M., \& Wakelam, V. 2013, ChRv, 113, 8710

Anglada, G., Sepúlveda, I., \& Gómez, J. F. 1997, A\&AS, 121, 255

Botschwina, P., \& Sebald, P. 1990, CP, 141, 311

Cernicharo, J. 2012, in European Conference on Laboratory Astrophysics, EAS Publications Series, Vol. 58, ed. C. Stehl, C. Joblin, \& L. d'Hendecourt (Cambridge: Cambridge Univ. Press), 251

Cernicharo, J., \& Guélin, M. 1987, A\&A, 176, 299

Cernicharo, J., Lefloch, B., Agúndez, M., et al. 2018, ApJL, 853, L22

Cernicharo, J., Marcelino, N., Roueff, E., et al. 2012, ApJL, 759, L43

Chaudhuri, R. K., Krishnamachari, S. L. N. G., \& Freed, K. F. 2006, JMoSt, 768, 119

Ding, Y.-H., Li, Z.-S., Huang, X.-R., \& Sun, C.-C. 2000, JChPh, 113, 1745

Ding, Y.-H., Li, Z.-S., Huang, X.-R., \& Sun, C.-C. 2001, JPCA, 105, 7085

Fray, N., Bénilan, Y., Cottin, H., Gazeau, M.-C., \& Crovisier, J. 2005, P\&SS, 53,1243

Gerry, M. C. L., Stroh, F., \& Winnewisser, M. 1990, JMoSp, 140, 147

Hily-Blant, P., Walmsley, M., Pineau Des Forêts, G., \& Flower, D. 2008, A\&A, 480, L5

Horn, M., Botschwina, P., \& Flügge, J. 1994, AcTC, 88, 1

Huckauf, A., Guarnieri, A., Heyl, Ä., et al. 1999, CPL, 303, 607

Huntress, W. T., Jr., Baldeschwieler, J. D., \& Ponnanperuma, C. 1969, Natur, 223, 468

Jiang, Z.-Y., Xu, X.-H., Wu, H.-S., et al. 2004, IJQC, 97, 876

Jolly, A., Cottini, V., Fayt, A., et al. 2015, Icar, 248, 340

Kawaguchi, K., Kasai, Y., Ishikawa, S.-I., \& Kaifu, N. 1995, PASJ, 47, 853

Kołos, R., \& Grabowski, Z. R. 2000, Ap\&SS, 271, 65

Kunde, V. G., Aikin, A. C., Hanel, R. A., et al. 1981, Natur, 292, 686

Lee, S. 1998, JMoSt, 427, 267

Loison, J. C., Hébrard, E., Dobrijevic, M., et al. 2015, Icar, 247, 218

Maier, G., Reisenauer, H. P., Eckwert, J., et al. 1992, Angew. Chem. Int. Ed. Engl., 31, 1218

Marcelino, N., Agúndez, M., Cernicharo, J., et al. 2018, A\&A, 612, L10

Marcelino, N., Cernicharo, J., Agúndez, M., et al. 2007, ApJL, 665, L127

Marcelino, N., Cernicharo, J., Roueff, E., et al. 2005, ApJ, 620, 308

Marcelino, N., Cernicharo, J., Tercero, B., \& Roueff, E. 2009, ApJL, 690, L27

McEwan, M. J., \& Anicich, V. G. 1995, JPhCh, 99, 12204

Oya, Y., Sakai, N., Watanabe, Y., et al. 2017, ApJ, 837, 174

Petrie, S. 1998, JPCA, 102, 7835

Petrie, S., Millar, T. J., \& Markwick, A. J. 2003, MNRAS, 341, 609

Petrie, S., \& Osamura, Y. 2004, JPCA, 108, 3623

Pickett, H. M., Poynter, R. L., Cohen, E. A., et al. 1998, JQSRT, 60, 883

Pratap, P., Dickens, J. E., Snell, R. L., et al. 1997, ApJ, 486, 862

Safrany, D. R., \& Jaster, W. 1968, JPhCh, 72, 3305

Soma, T., Sakai, N., Watanabe, Y., \& Yamamoto, S. 2018, ApJ, 854, 116

Tafalla, M., Myers, P. C., Mardones, D., \& Bachiller, R. 2000, A\&A, 359, 967

Whyte, A. R., \& Phillips, L. F. 1983, CPL, 98, 590

Winnewisser, M., Seibert, J. W. G., \& Yamada, K. M. T. 1992, JMoSp, 153, 635

Yang, D. L., Yu, T., Lin, M. C., \& Melius, C. F. 1992, JChPh, 97, 222 